\title{
Validation of biomarkers associated with 5-fluorouracil and thymidylate synthase in colorectal cancer
}

\author{
YAGUANG XI $^{1 *}$, ANDREA FORMENTINI ${ }^{2 *}$, GO NAKAJIMA $^{1}$, MARKO KORNMANN $^{2}$ and JINGFANG JU ${ }^{1}$ \\ ${ }^{1}$ The Mitchell Cancer Institute, University of South Alabama, Mobile, AL 36688, USA; \\ ${ }^{2}$ Department of General, Visceral and Transplantation Surgery, University of Ulm, 89075 Ulm, Germany
}

Received July 9, 2007; Accepted August 13, 2007

\begin{abstract}
Previous studies from our laboratory have identified a number of genes associated with chemosensitivity to 5fluorouracil (5-FU) using an in vitro colon cancer cell line model. In this study, the in vivo significance of several marker genes in terms of prognostic potential was evaluated using colorectal cancer patient samples. Eight marker genes were selected based on their functional roles and significant fold changes in expression. They are SERTA domain containing 1 (SEI1), ribonucleotide reductase M2 polypeptide (RRM2), origin recognition complex, subunit 6 homolog-like (ORC6L), eukaryotic translation initiation factor 4E (EIF4E), thymidylate synthase (TS), SET and MYND domain containing 3 (SMYD3), Dickkopf homolog 4, and methyl-CpG binding domain protein 4 (MBD4). Forty-eight snap frozen clinical colorectal samples (24 normal and 24 paired colorectal cancer patient samples) were selected with detailed clinical followup information. cDNAs were synthesized and the expression levels of marker genes were quantified via qRT-PCR analysis. The statistical significance of these markers for disease prognosis was evaluated using the two-tailed paired Wilcoxon test. Survival curves were plotted according to the method of Kaplan-Meier and compared using the log-rank test. Based on the quantitative expression analysis, RRM2 ( $\mathrm{p}=0.0001$; 95\% CI, 2.0-4.5), ORC6L ( $\mathrm{p}=0.0001 ; 95 \%$ CI, 1.8-4.6), EIF4E $(\mathrm{p}=0.0002 ; 95 \% \mathrm{CI}, 0.3-0.9)$, TS ( $\mathrm{p}=0.0005 ; 95 \% \mathrm{CI}, 0.7-2.2)$ and SMYD3 ( $\mathrm{p}=0.0001 ; 95 \% \mathrm{CI}, 0.8-1.5)$ were overexpressed in tumor tissues. However, the expression of SEI1 was decreased in tumors ( $\mathrm{p}=0.02 ; 95 \% \mathrm{CI}, 0.1-1.3)$, consistent with the function of SEI1 as a potential tumor suppressor. KaplanMeier survival analysis indicated that MBD4 is a significant prognostic factor for patient survival ( $\mathrm{p}=0.03$ ). MBD4 was a
\end{abstract}

Correspondence to: Dr Jingfang Ju, Cancer Genomics Laboratory, The Mitchell Cancer Institute, University of South Alabama, Mobile, AL 36688, USA

E-mail: jju@usouthal.edu

${ }^{*}$ Contributed equally

Key words: colorectal cancer, methyl-CpG binding domain protein 4, survival, biomarker key protein involved in DNA methylation. The expression of TS was associated with tumor stage as it had a significantly higher expression level in UICC stage I and II compared to stage IV patients $(\mathrm{p}=0.03)$. MBD4 may be a potential novel prognostic marker for predicting patient survival for colorectal cancer.

\section{Introduction}

Despite adjuvant 5-fluorouracil (5-FU)-based therapy, nearly $30 \%$ of patients with advanced colorectal cancer develop recurrence. In order to target treatment of colorectal cancer more effectively in the future, it will be particularly important to identify patients who are at risk of recurrence receiving 5-FU-based therapy. Such patients could be treated with some of the recently approved compounds such as bevacizumab, cetuximab, oxaliplatin and irinotecan. Moreover, nonresponders to 5-FU will have the option to be treated firstly with non-fluoropyrimidine-based drugs to improve response and to avoid unnecessary toxic side-effects. It has been shown that patients with 5-FU refractory colorectal cancer respond to irinotecan (1). Cetuximab was also demonstrated to have significant activity when given alone or in combination with irinotecan in patients with irinotecan-refractory colorectal cancer (2). Therefore, identification of prognostic markers for colorectal cancer treatment is important to optimize the treatment plan of individual patients with both early and advanced disease. Extensive efforts and progress have been made in the past decade by utilizing both high-throughput genomics approaches and highly quantitative measurements such as real-time qRT-PCR analysis particularly for the fluropyrimidine-based drugs such as 5-FU. A number of genes have been shown to be closely associated with response to 5-FU-based therapy, including cyclin D, TS, thymidine phosphorylase, and dihydropyrimidine dehydrogenase $(3,4)$. In these studies, patients with a low expression level of TS tended to respond better than patients with a high TS level. However, in other studies the opposite results were observed $(5-8)$. It is clear that additional markers are clearly needed to optimize the prognostic power for individual patients.

To further expand the candidate genes associated with 5-FU treatment and TS overexpression, our group developed a systems biology approach to systematically discover genes at both transcriptional and post-transcriptional levels using a pair of colon cancer cell lines (9). As a result, a number of 
genes were discovered to be closely associated with the overexpression of 5-FU target enzyme thymidylate synthase (TS) and 5-FU treatment using a pair of colon cancer cell lines at multi-levels of gene regulation by high-throughput gene expression analysis (9). In particular, a number of potential novel post-transcriptional regulated genes have been identified with this unique approach. This study provided us with a new pool of candidate genes for further in vivo evaluation. In the present study, we selected 8 genes from our comprehensive gene expression profiling analysis (9): SERTA domain containing 1 (SEI1), ribonucleotide reductase M2 polypeptide (RRM2), origin recognition complex, subunit 6 homolog-like (ORC6L), eukaryotic translation initiation factor 4E (EIF4E), thymidylate synthase (TS), SET and MYND domain containing 3 (SMYD3), Dickkopf homolog 4 (Dkk4) and methyl-CpG binding domain protein 4 (MBD4). The selection was based on their critical biological functions and significant expression differences in response to 5-FU treatment and TS overexpression (10-16). To further validate the in vivo significance of some of the highly significant markers that may be associated with chemoresponse to 5-FU-based therapy, a group of colorectal cancer patient specimens were selected. We have confirmed that several marker genes are differentially expressed in colorectal clinical specimens. The expression of MBD4 was associated with patient survival. Given the absent expression of Dkk4 in many of the normal colorectal samples, it may be a good candidate gene for anti-tumor drug discovery.

\section{Materials and methods}

Patients and samples. A total of 48 snap frozen colorectal patient specimens were selected (24 paired normal and tumor). These patients had undergone surgical resection of primary colorectal adenocarcinoma at the Department of General, Visceral and Transplantation Surgery, University of Ulm, Germany. Patient consent forms were obtained from every patient according to the institutional regulations. The characteristics of these patients are shown in Table I. Some of these patients were treated with adjuvant 5-FU-based chemotherapy and others were treated with palliative highdose 5-FU/FA (Table I).

RNA isolation and cDNA synthesis. Total RNAs were isolated using a previously published protocol (17). In brief, TRIzol reagent (Invitrogen, CA) was used to isolate total RNA from snap frozen tissues. RNA was treated with DNase I (Promega, WI). The integrity of total RNA was determined by $1 \%$ formaldehyde-agarose gel electrophoresis. cDNA synthesis was carried out with the cDNA synthesis kit (Applied Biosystems Inc, CA) using $1 \mu \mathrm{g}$ of total RNA as the template and random primers at $25^{\circ} \mathrm{C}$ for $10 \mathrm{~min}$ and $37^{\circ} \mathrm{C}$ for $2 \mathrm{~h}$ of reverse transcription.

Real-time qRT-PCR analysis. Real-time qRT-PCR analysis was performed on selected genes based on a previously published procedure (17). Real-time qRT-PCR analysis was performed on the experimental mRNAs $(n=3)$. The PCR primers and probes for EIF4E, MBD4, SEI1, SMYD3, TS, ORC6L, Dkk4, RRM2, and internal control gene GAPDH were purchased from Applied Biosystems Inc. qRT-PCR was performed on
Table I. Clinical features of the 24 patients in the series studied.

\begin{tabular}{|c|c|c|}
\hline Characteristics & Frequency & Percentage \\
\hline \multicolumn{3}{|l|}{ Age (years) } \\
\hline Mean (range) & $62(30-93)$ & \\
\hline \multicolumn{3}{|l|}{ Gender } \\
\hline Male & 14 & 58.3 \\
\hline Female & 10 & 41.7 \\
\hline \multicolumn{3}{|l|}{ Anatomic site } \\
\hline Ascending colon & 3 & 12.5 \\
\hline Transverse colon & 2 & 8.3 \\
\hline Descending colon & 4 & 16.7 \\
\hline Sigmoid colon & 3 & 12.5 \\
\hline Rectum & 12 & 50.0 \\
\hline \multicolumn{3}{|l|}{ Histology } \\
\hline Adenocarcinoma & 24 & 100 \\
\hline \multicolumn{3}{|l|}{ UICC stage } \\
\hline I & 4 & 16.7 \\
\hline II & 4 & 16.7 \\
\hline III & 8 & 33.3 \\
\hline IV & 8 & 33.3 \\
\hline \multicolumn{3}{|l|}{ Survival (months) } \\
\hline Mean (range) & $30(0-75)$ & \\
\hline $0-20$ & 3 & 12.5 \\
\hline $20-50$ & 20 & 83.3 \\
\hline$>50$ & 1 & 4.2 \\
\hline
\end{tabular}

an $\mathrm{ABI} 7500 \mathrm{HT}$ instrument under the following conditions: 40 cycles of $50^{\circ} \mathrm{C}$ for $2 \mathrm{~min}, 95^{\circ} \mathrm{C}$ for $10 \mathrm{~min}, 95^{\circ} \mathrm{C}$ for $15 \mathrm{sec}$ and $60^{\circ} \mathrm{C}$ for $1 \min (n=3)$.

Statistical analysis. Expression analysis of each gene was performed between each pair of normal and tumor samples to identify any potential tumor markers by the Wilcoxon test using MedCalc ${ }^{\circledR}$ for Windows version 8.1.1.0 (MedCalc software, Mariakerke, Belgium). The expression of these genes was also evaluated by grouping samples to different stages of the disease using the paired Wilcoxon test. Patient survival data were analyzed by the method of Kaplan and Meier. The logrank test was used to evaluate the significance for patient survival. For important prognostic factors, the hazard ratio with corresponding 95\% CI and the P-value were calculated.

\section{Results}

Overexpressed genes in colorectal cancer that may serve as a tumor marker. First the expression levels of eight selected genes (EIF4E, MBD4, SEI1, SMYD3, TS, ORC6L, Dkk4, and RRM2) were quantified by real-time qRT-PCR analysis approach. The level of expression of each individual gene was normalized with the house-keeping gene GAPDH. Next we 
A

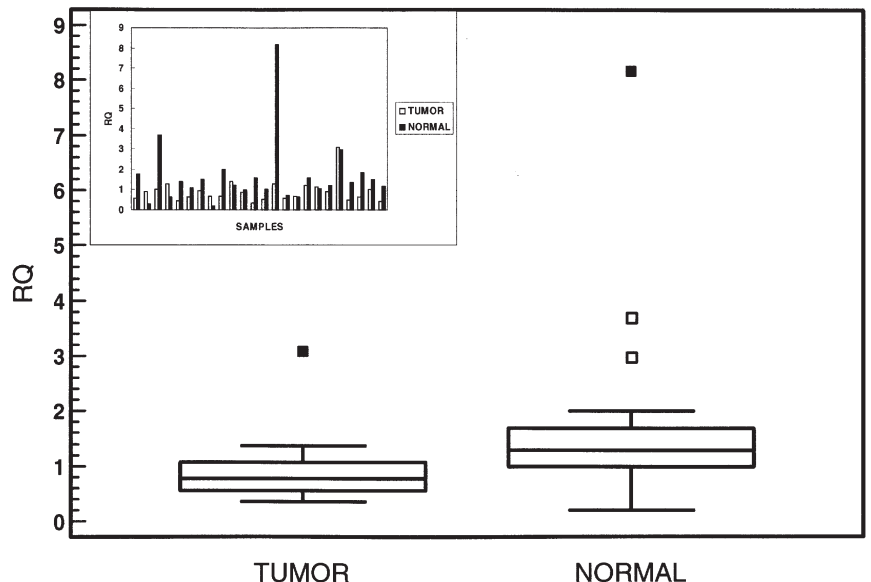

B

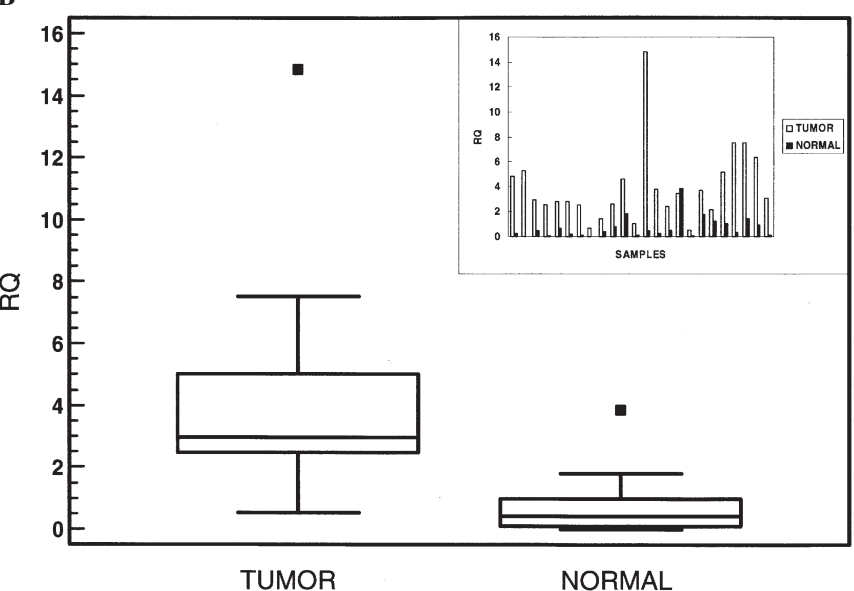

C

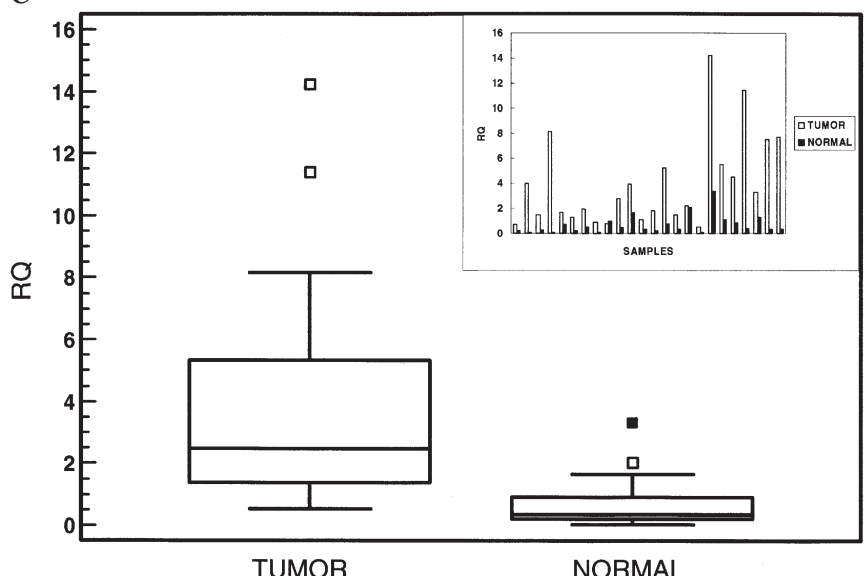

D

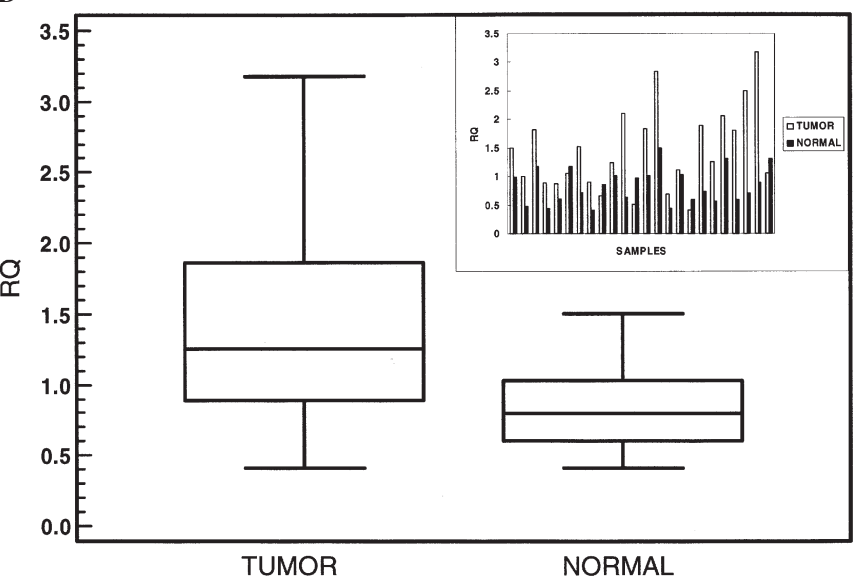

$\mathbf{E}$

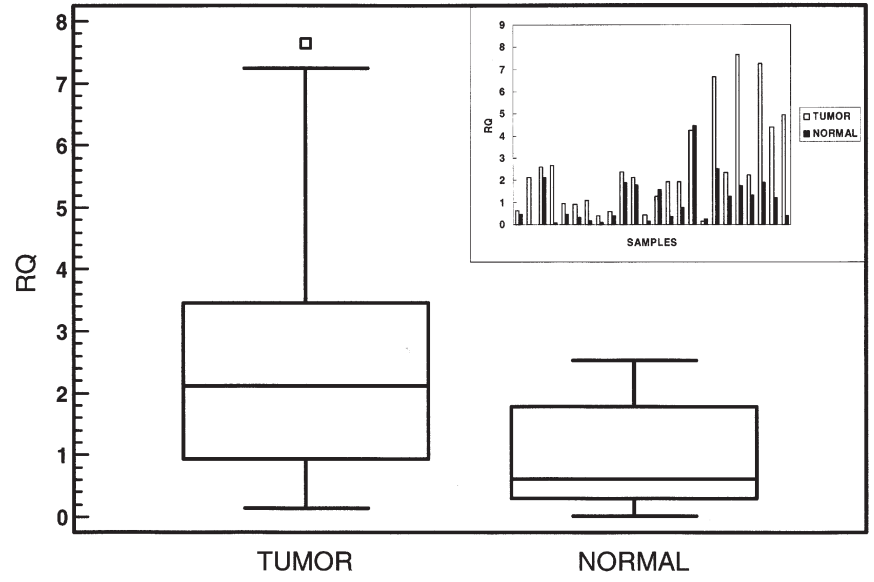

F

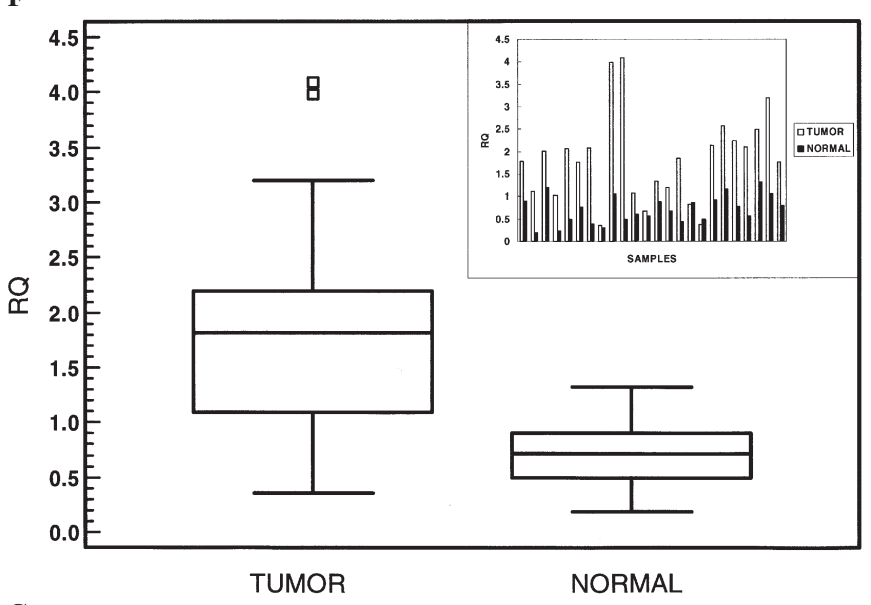

G

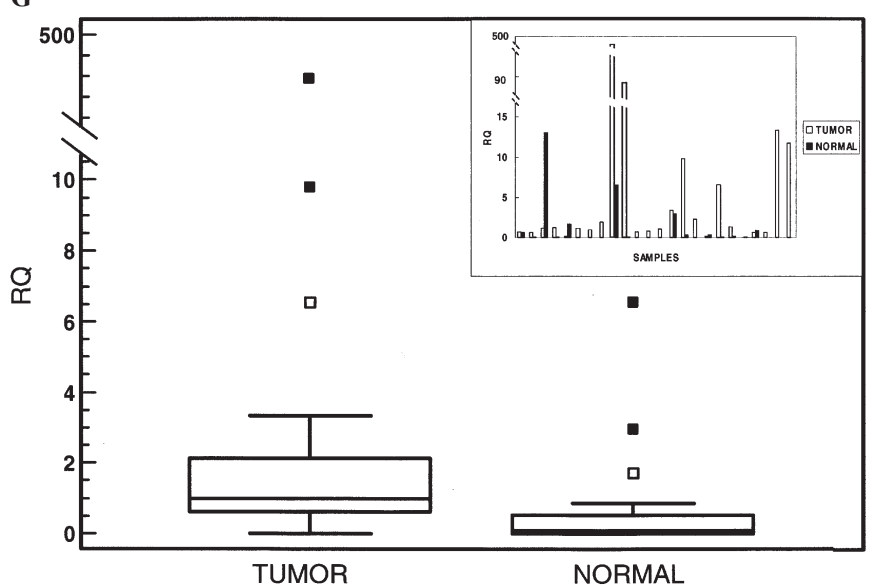

H

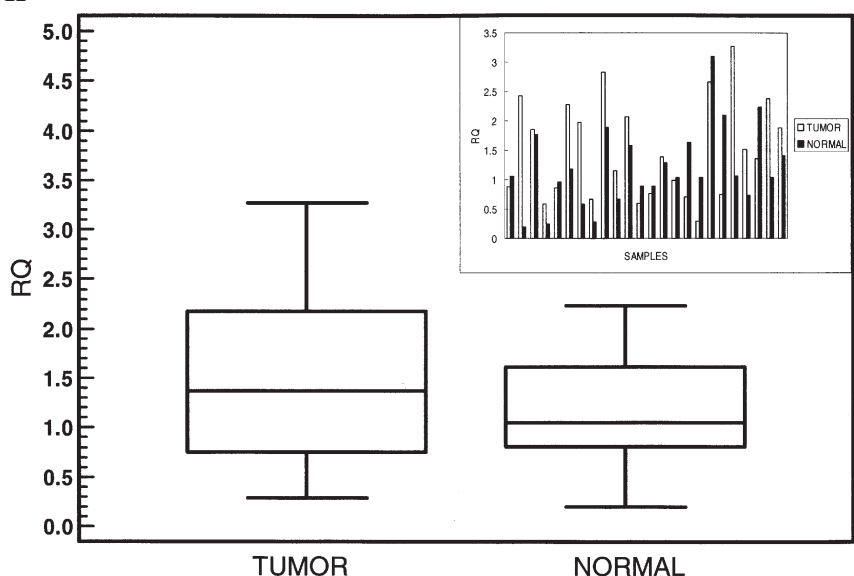

Figure 1. Expression analysis of SEI1 (A), RRM2 (B), ORC6L (C), EIF4E (D), TS (E), SMYD4 (F), Dkk4 (G), and MBD4 (H) in colorectal cancer. Gene expression values were expressed as $\triangle \triangle \mathrm{CT}$ normalized with the $G A P D H$ internal reference control gene $(\mathrm{n}=3)$. 


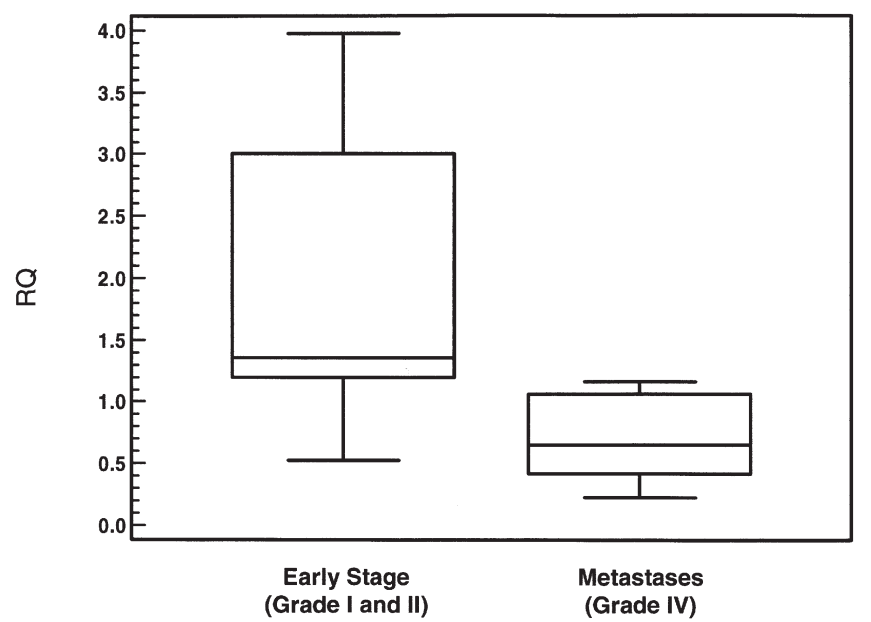

Figure 2. Expression analysis of TS in early stage (I, II) and metastatic (IV) colorectal cancer. A significant difference was observed in TS expression between stage I and II vs. stage IV ( $p=0.003)$.

compared the expression levels of each candidate gene from tumor samples to the corresponding normal counterparts. Surprisingly, most of the selected marker genes were significantly different between the tumors and corresponding normal samples. As shown in Fig. 1, the average expression of SEI1 (Fig. 1A) in normal samples was nearly 2-fold ( $\mathrm{p}=0.02$, $95 \% \mathrm{CI}$ ) higher than in tumor samples. RRM2 was highly overexpressed in tumors compared to normal samples (5.6fold with $\mathrm{p}=0.0001,95 \% \mathrm{CI}$ ) (Fig. 1B). ORC6L was also significantly overexpressed by nearly 6 -fold $(p=0.0001)$ in tumors compared to normal samples (Fig. 1C). The level of EIF4E was higher in tumors (average value, 1.45) vs. normal samples (average value, 0.84) (Fig. 1D). The 5-FU target enzyme TS was overexpressed by $>2$-fold in tumors compared to normal specimens (Fig. 1E). The average expression of SMYD3 was 1.84 which is $>2$-fold higher in tumors compared to normal samples with an average value of $0.71(\mathrm{p}=0.0001)$ (Fig. 1F). The expression of Dkk4 was completely missing from 10 normal samples and the 14 remaining normal samples also had a lower level of Dkk4 expression (by 8-fold) compared to tumor samples (Fig. 1G). However, the expression of MBD4 was not different between normal and tumor samples $(\mathrm{p}=0.15)$ (Fig. 1H).

High TS levels were associated with disease progression. We analyzed the potential association of all eight genes with disease progression in terms of disease stage. The expression levels of TS in late-stage tumors (UICC IV) were significantly higher (>2-fold) than in early-stage tumors (UICC I, II) (Fig. 2).

High MBD4 transcript levels were associated with a better prognosis. Kaplan-Meier survival curves were generated based on the expression level of eight markers. Among these genes, the expression level of MBD4 was strongly associated with patient survival ( $\mathrm{p}=0.03$ ). Patients with a high MBD4 level had the best disease-specific survival from the start of adjuvant treatment to tumor-related death (Fig. 3A). Although SEI1 expression did not correlate very strongly with patient survival
A

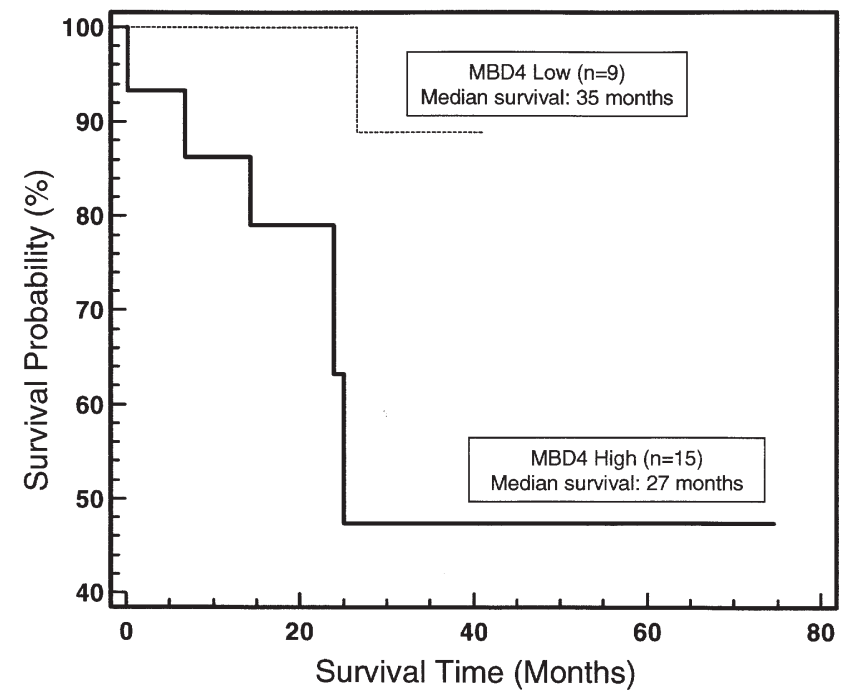

B

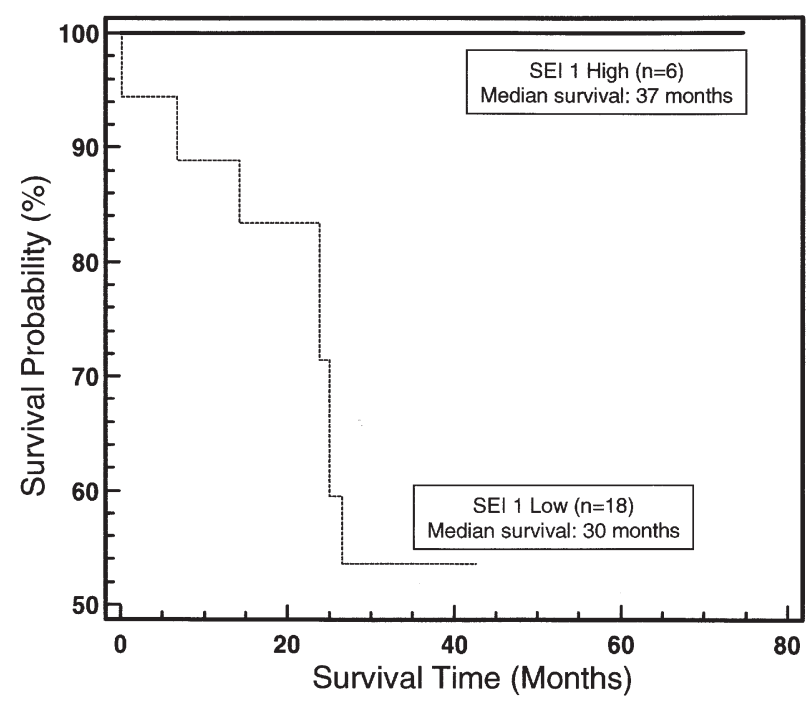

Figure 3. Kaplan-Meier survival analysis according to MBD4 ( $\mathrm{p}=0.03)$ (A) and SEI1 ( $\mathrm{p}=0.08)(\mathrm{B})$ expression levels in colorectal cancer patients who received surgery and 5-FU-based therapy (log-rank p-values).

$(\mathrm{p}=0.08)$, all six patients with higher SEI1 expression were still alive at the evaluation time point.

\section{Discussion}

In this study, the in vivo significance of previously identified genes that are associated with TS overexpression and 5-FU treatment were further evaluated using a panel of selected colorectal specimens. Our rationale is that it is difficult to generate reliable and consistent markers using a global expression profiling approach. It has been recently documented by Ein-Dor et al that thousands of samples are needed to generate a robust gene list for predicting outcome in cancer (18). Our approach is based on discovered marker genes associated with chemosensitivity using a well-defined colon cancer model HCT-C18 (TS-) and HCT-C18 (TS ${ }^{+}$) (9). This will allow us to further reduce the number of genes to a limited number for further validation of their usefulness using clinical samples. Twenty-four uniquely paired normal 
and corresponding colorectal tumors were chosen for the comparison. Among these 24 patients, seven received adjuvant 5-FU-based chemotherapy and six received palliative highdose 5-FU/FA treatment. We were surprised to see that the majority of the selected genes were significantly overexpressed in tumors (with the exception of SEI1) compared to their normal counterparts based on the real-time qRT-PCR analysis. EIF4E, a key translational initiation factor, was expressed at a higher level in tumors. It has been shown that EIF4E plays a key role in response to chemotherapy. EIF4E is one of the key translation initiation factors and has been shown to play a key role in chemoresistance and mTOR pathway (19-21). Our finding is consistent with the fact that elevated EIF4E expression in colorectal tumors contributes to chemoresistance. In contrast, the expression of SEI1 was lower in tumors than normal samples. SEI1 has been shown to play a key role in G1/S transition and activate transcription of p21 independent of p53 (22). SEI proteins possess an intrinsic transactivation activity, interact with the co-activator CREB-binding protein, and cooperate synergistically with the ING family of chromatin-associated proteins to stimulate the transactivation function of p53. In this study, Watanabe-Fukunaga et al found that the doxycycline-induced expression of SEI proteins results in activation of the p21 gene and inhibition of cell growth, but the growth arrest was not suppressed by the siRNA-mediated knockdown of the endogenous p53 protein. These results indicate that the SEI family of nuclear proteins regulates p53 transcriptional activity and a p53-independent signaling pathway leading to growth inhibition. Decreased expression of SEI1 may also contribute to the drug resistance mechanism in colorectal cancer. Kaplan-Meier survival analysis showed that six patients with higher expression levels of SEI1 lived longer than those in the low expression group (Fig. 3B).

Another important gene is ORC6L, which was overexpressed in tumor samples in our study (Fig. 1C). ORC6L is a protein involved in the DNA replication complex and acts as an essential gene that coordinates chromosome replication and segregation with cytokinesis $(23,24)$. The overexpression of ORC6L in colorectal tumors may indicate a strong association with the disease development. Another striking finding is that the expression of Dkk4 was completely undetectable in 10 of the 24 normal samples, the remaining 14 normal samples also had 8-fold lower expression of Dkk4 compared to tumors (Fig. 1G). This is the first report that in many cases, the expression of Dkk4 is missing in normal colorectal samples. Dkk4 is one of the key antagonists for Wnt/ß-catenin signaling pathway (25). Dkk4 would be an ideal target because it is not expressed in normal colon tissue based on our data.

RRM2 and TS were also overexpressed in tumors compared to normal colorectal samples. RRM2 and TS belong to the pyrimidine metabolic pathway. The expression of RRM2 has been shown to play a key role in chemosensitivity (26-28). However, TS was not associated with survival in these samples. The TS level was significantly higher in UICC stage IV metastatic tumors than in primary tumors, indicting that it may be associated with disease progression. Our finding is consistent with previous results reported by Johnston et al (3).
Finally, despite a relative short-time follow-up, MBD4 was a significant marker gene that was associated with patient survival. It has been found that it may suppress neoplasia. MBD4 was characterized as a thymine DNA glycosylase that interacts with the mismatch repair (MMR) protein MLH1. The in vivo function of MBD4 is to decrease the mutability of methyl-CpG sites in the genome and mice deficient in MBD4 show increased intestinal tumorigenesis. It was demonstrated that mice deficient for MBD4 showed significantly reduced apoptotic responses following treatment with a range of cytotoxic agents such as 5-FU, $\gamma$-irradiation and cisplatin. This leads to increased clonogenic survival in vivo in Mbd4 (-/-) mice following exposure to either 5-FU or cisplatin. These results reported by other groups provide further support for our results that MBD4 is a potential indicator for patient survival.

In summary, we have confirmed that several of the marker genes are differentially expressed in colorectal clinical specimens. MBD4 was significantly associated with patient survival. Given the absent expression of Dkk4 in many of the normal colorectal samples, it may be a good candidate gene for antitumor drug discovery. Further studies are clearly needed to validate the prognostic utility of MBD4 using a larger cohort of clinical samples.

\section{Acknowledgements}

This project was supported by the USA-Mitchell Cancer Institute Start-Up fund (J. Ju).

\section{References}

1. Van Cutsem E, Dirix L, Van Laethem JL, Van Belle S, Borner M, Gonzalez Baron M, Roth A, Morant R, Joosens E, Gruia G, Sibaud D and Bleiberg H: Optimisation of irinotecan dose in the treatment of patients with metastatic colorectal cancer after 5-FU failure: results from a multinational, randomised phase II study. Br J Cancer 92: 1055-1062, 2005.

2. Cunningham D, Humblet Y, Siena S, Khayat D, Bleiberg H, Santoro A, Bets D, Mueser M, Harstrick A, Verslype C, Chau I and Van Cutsem E: Cetuximab monotherapy and cetuximab plus irinotecan in irinotecan-refractory metastatic colorectal cancer. N Engl J Med 351: 337-345, 2004.

3. Johnston PG, Fisher ER, Rockette HE, Fisher B, Wolmark N, Drake JC, Chabner BA and Allegra CJ: The role of thymidylate synthase expression in prognosis and outcome of adjuvant chemotherapy in patients with rectal cancer. J Clin Oncol 12: 2640-2647, 1994.

4. Salonga D, Danenberg KD, Johnson M, Metzger R, Groshen S, Tsao-Wei DD, Lenz HJ, Leichman CG, Leichman L, Diasio RB and Danenberg PV: Colorectal tumors responding to 5fluorouracil have low gene expression levels of dihydropyrimidine dehydrogenase, thymidylate synthase, and thymidine phosphorylase. Clin Cancer Res 6: 1322-1327, 2000.

5. Takenoue T, Nagawa H, Matsuda K, Fujii S, Nita ME, Hatano K, Kitayama J, Tsuruo T and Muto T: Relation between thymidylate synthase expression and survival in colon carcinoma, and determination of appropriate application of 5-fluorouracil by immunohistochemical method. Ann Surg Oncol 7: 193-198, 2000

6. Edler D, Glimelius B, Hallstrom M, Jakobsen A, Johnston PG, Magnusson I, Ragnhammar P and Blomgren H: Thymidylate synthase expression in colorectal cancer: a prognostic and predictive marker of benefit from adjuvant fluorouracil-based chemotherapy. J Clin Oncol 20: 1721-1728, 2002.

7. Yamachika T, Nakanishi H, Inada K, Tsukamoto T, Kato T, Fukushima M, Inoue $\mathrm{M}$ and Tatematsu $\mathrm{M}$ : A new prognostic factor for colorectal carcinoma, thymidylate synthase, and its therapeutic significance. Cancer 82: 70-77, 1998. 
8. Kornmann M, Schwabe W, Sander S, Kron M, Strater J, Polat S, Kettner E, Weiser HF, Baumann W, Schramm H, Hausler P, Ott K, Behnke D, Staib L, Beger HG and Link KH: Thymidylate synthase and dihydropyrimidine dehydrogenase mRNA expression levels: predictors for survival in colorectal cancer patients receiving adjuvant 5-fluorouracil. Clin Cancer Res 9: 4116-4124, 2003.

9. Xi Y, Nakajima G, Schmitz JC, Chu E and Ju J: Multi-level gene expression profiles affected by thymidylate synthase and 5-fluorouracil in colon cancer. BMC Genomics 7: 68, 2006.

10. Rosenwald IB, Chen JJ, Wang S, Savas L, London IM and Pullman J: Upregulation of protein synthesis initiation factor eIF-4E is an early event during colon carcinogenesis. Oncogene 18: 2507-2517, 1999.

11. Sansom OJ, Zabkiewicz J, Bishop SM, Guy J, Bird A and Clarke AR: MBD4 deficiency reduces the apoptotic response to DNA-damaging agents in the murine small intestine. Oncogene 22: 7130-7136, 2003.

12. Sugimoto M, Nakamura T, Ohtani N, Hampson L, Hampson IN, Shimamoto A, Furuichi Y, Okumura K, Niwa S, Taya Y and Hara E: Regulation of CDK4 activity by a novel CDK4-binding protein, p34(SEI-1). Genes Dev 13: 3027-3033, 1999.

13. Mao B and Niehrs C: Kremen2 modulates Dickkopf2 activity during Wnt/LRP6 signaling. Gene 302: 179-183, 2003.

14. Diep DB, Hoen N, Backman M, Machon O and Krauss S: Characterisation of the Wnt antagonists and their response to conditionally activated Wnt signalling in the developing mouse forebrain. Brain Res Dev Brain Res 153: 261-270, 2004.

15. Appelt K, Bacquet RJ, Bartlett CA, Booth CL, Freer ST, Fuhry MA, Gehring MR, Herrmann SM, Howland EF, Janson CA, et al: Design of enzyme inhibitors using iterative protein crystallographic analysis. J Med Chem 34: 1925-1934, 1991.

16. Danenberg PV: Thymidylate synthetase - a target enzyme in cancer chemotherapy. Biochim Biophys Acta 473: 73-92, 1977.

17. Ju J, Huang C, Minskoff SA, Mayotte JE, Taillon BE and Simons JF: Simultaneous gene expression analysis of steadystate and actively translated mRNA populations from osteosarcoma MG-63 cells in response to IL-1alpha via an open expression analysis platform. Nucleic Acids Res 31: 5157-5166, 2003.
18. Ein-Dor L, Zuk O and Domany E: Thousands of samples are needed to generate a robust gene list for predicting outcome in cancer. Proc Natl Acad Sci USA 103: 5923-5928, 2006.

19. McCormick F: Cancer: survival pathways meet their end. Nature 428: 267-269, 2004

20. Wendel HG, De Stanchina E, Fridman JS, Malina A, Ray S, Kogan S, Cordon-Cardo C, Pelletier J and Lowe SW: Survival signalling by Akt and eIF4E in oncogenesis and cancer therapy. Nature 428: 332-337, 2004

21. Wendel HG and Lowe SW: Reversing drug resistance in vivo. Cell Cycle 3: 847-849, 2004.

22. Watanabe-Fukunaga R, Iida S, Shimizu Y, Nagata S and Fukunaga R: SEI family of nuclear factors regulates p53dependent transcriptional activation. Genes Cells 10: 851-860, 2005.

23. Killian A, Le Meur N, Sesboue R, Bourguignon J, Bougeard G, Gautherot J, Bastard C, Frebourg T and Flaman JM: Inactivation of the RRB1-Pescadillo pathway involved in ribosome biogenesis induces chromosomal instability. Oncogene 23: 8597-8602, 2004

24. Prasanth SG, Prasanth KV and Stillman B: Orc6 involved in DNA replication, chromosome segregation, and cytokinesis. Science 297: 1026-1031, 2002.

25. Boerboom D, White LD, Dalle S, Courty J and Richards JS: Dominant-stable beta-catenin expression causes cell fate alterations and Wnt signaling antagonist expression in a murine granulosa cell tumor model. Cancer Res 66: 1964-1973, 2006.

26. Bergman AM, Eijk PP, Ruiz van Haperen VW, Smid K, Veerman G, Hubeek I, van den Ijssel P, Ylstra B and Peters GJ: In vivo induction of resistance to gemcitabine results in increased expression of ribonucleotide reductase subunit M1 as the major determinant. Cancer Res 65: 9510-9516, 2005.

27. Zhou B, Mo X, Liu X, Qiu W and Yen Y: Human ribonucleotide reductase M2 subunit gene amplification and transcriptional regulation in a homogeneous staining chromosome region responsible for the mechanism of drug resistance. Cytogenet Cell Genet 95: 34-42, 2001

28. Zhou B and Yen Y: Characterization of the human ribonucleotide reductase M2 subunit gene; genomic structure and promoter analyses. Cytogenet Cell Genet 95: 52-59, 2001. 